\title{
An in vivo evaluation of Brilliant Blue $G$ in animals and humans
}

\author{
M Remy, ${ }^{1}$ S Thaler, ${ }^{2}$ R G Schumann, ${ }^{1}$ C A May, ${ }^{3}$ M Fiedorowicz, ${ }^{2,4} \mathrm{~F}$ Schuettauf, ${ }^{2}$ \\ M Grüterich, ${ }^{1}$ S G Priglinger, ${ }^{1}$ M M Nentwich, ${ }^{1}$ A Kampik, ${ }^{1}$ C Haritoglou'
}

${ }^{1}$ Department of Ophthalmology, Ludwig-Maximilians-University, Munich, Germany: ${ }^{2}$ Center for Ophthalmology, University Eye Hospital, Tübingen, Germany:

${ }^{3}$ Department of Anatomy, Technische Universität, Dresden, Germany; ${ }^{4}$ Department of Experimental Pharmacology, PAS Medical Research Center, Warsaw, Poland

Correspondence to: Dr C Haritoglou, Department of Ophthalmology, LudwigMaximilians-University, Mathildenstr. 8, 80336 Munich. Germany; christos.haritoglou@ med.uni-muenchen.de

MR and ST contributed equally to this work.

Accepted 10 May 2008

\section{ABSTRACT}

Background/Aims: To evaluate the retinal toxicity of Brilliant Blue G (BBG) following intravitreal injection in rat eyes and examine the biocompatibility and the staining properties in humans.

Methods: BBG was injected into the 11 rat eyes to evaluate toxic effects with balanced salt solution (BSS) serving as control. Retinal toxicity was assessed by retinal ganglion cell (RGC) counts and by light microscopy 7 days later. In addition, BBG was applied during vitrectomy for macular hole $(\mathrm{MH})(\mathrm{n}=15)$ or epiretinal membranes (ERM) $(n=3)$ in a prospective, non-comparative consecutive series of patients. Before and after surgery, all patients underwent a complete clinical examination including measurement of best corrected visual acuity (VA) and intraocular pressure, perimetry, fundus photography and optical coherence tomography. Patients were seen 1 day before surgery and then in approximately four weeks intervals.

Results: No significant reduction in RGC numbers and no morphological alterations were noted. A sufficient staining of the internal limiting membrane (ILM) was seen in patients with $\mathrm{MH}$, while the staining pattern in ERM cases was patchy, indicating that parts of the ILM were peeled off along with the ERM in a variable extent. All MHs could be closed successfully. VA improved in 10 eyes (56\%; 8/ $15 \mathrm{MH}$ patients, 2/3 ERM patients), was unchanged in four eyes (22\%; all MH patients) and was reduced in four eyes (22\%; 3/15 MH, 1/3 ERM). No toxic effects attributable to the dye were noted during patient followup. The ultrastructure of tissue harvested during surgery was unremarkable.

Conclusion: Brilliant Blue provides a sufficient and selective staining of the ILM. No retinal toxicity or adverse effects related to the dye were observed in animal and human studies. The long-term safety of this novel dye will have to be evaluated in larger patient series and a longer follow-up.

Vital dyes have become very popular to visualise barely visible structures such as the internal limiting membrane (ILM) during macular hole (MH) surgery using Indocyanine Green (ICG), ${ }^{1}$ epiretinal membranes (ERM) using Trypan Blue $(\mathrm{TB})^{2}$ and the vitreous, which can be visualised using Bromophenol Blue. ${ }^{3}$ The main advantages of ICG were the good contrast provided and its selective staining properties. However, at present the "off label" intravitreal application of ICG has become a subject of ongoing discussion, as toxic effects of the dye leading to functional impairment of the patients cannot be ruled out. ${ }^{4-7}$ TB appears to be safe for intraocular application, but provides a far less pronounced staining of the ILM and lacks the selective staining properties of ICG and requires a fluid-air exchange to achieve high enough concentration on the retinal surface. The latter may be overcome by special TB preparations. ${ }^{8}$ Recently, Enaida and co-workers introduced Brilliant Blue G (BBG) as an alternative for ILM peeling. ${ }^{9-11}$ The purpose of this investigation was to assess potential retinal toxicity of BBG in a well-established ${ }^{12}$ animal model and to evaluate the staining properties and safety profile in a prospective series of patients undergoing $\mathrm{MH}$ and ERM surgery.

\section{MATERIALS AND METHODS}

\section{Animal study}

\section{Animals}

All experiments were performed in accordance with guidelines for animal care in the European Community and those of the Association for Research in Vision and Ophthalmology. Male Brown Norway rats (Charles River, Sulzfeld, Germany) with a body weight of 120-160 g were used. Rats were housed under a $12 \mathrm{~h}$ light-dark cycle.

Intravitreal dye injections

Eleven adult male Brown Norway rats were anaesthetised with an intraperitoneal injection of chloral hydrate $(7 \%, 6 \mathrm{ml} / \mathrm{kg}$ body weight). Intravitreal BBG (Brilliant Peel; Fluoron $\mathrm{GmbH}$, Neu-Ulm, Germany/306 mOsm/ $\mathrm{kg} \quad \mathrm{H}_{2} \mathrm{O}$; $\mathrm{pH}=7.52$ ) injections were performed using a heat-pulled glass capillary connected to a microsyringe (Drummond Scientific, Broomall, PA), under direct observation through the microscope. Eyes with lens damage were excluded from the experiments and not used thereafter. Single injections of $2 \mu \mathrm{l}$ of BBG $(0.25 \%)$ were performed, reaching a final intraocular $\mathrm{BBG}$ concentration of $0.025 \%$ (estimated for an average rat eye intravitreal volume of $20 \mu \mathrm{l}$ ). Contralateral eyes served as control eyes and were injected with balanced salt solution (BSS).

Retrograde labelling and quantification of retinal ganglion cells (RGC)

RGC survival was assessed as described previously. ${ }^{12}$ In brief, anaestetised rats were placed into a stereotaxic frame (TSE systems, Bad Homburg, Germany). A portion of the skull and the meninges were removed, exposing the neocortex overlying the tectum on the side contralateral of the lesioned eye. Three injections of the fluorescent tracer Fluorogold (Hydroxystilbamidine methanesulfonate, Molecular Probes, Eugene, OR) were made over a period of 
2 min each targeting all layers of both superior colliculi. Two days later, the animals were sacrificed with a choral hydrate overdose. Eyes were enucleated, retinas dissected, flat-mounted, and fixed in $3 \%$ paraformaldehyde (PFA) for $30 \mathrm{~min}$. Visualisation was performed immediately under fluorescent microscope. Before enucleation the cornea was marked at the 12 o'clock position for orientation in retinal quadrants. The RGC-counting was carried out in 12 distinct areas of $62500 \mu \mathrm{m}^{2}$. Images obtained using a digital imaging system (ImagePro 3.0, Media Cybernetics, Silver Spring, MD) connected to a microscope were coded and analysed in a masked fashion. Labelled cells were defined as surviving. Counts are expressed as the cell density (cells per square millimetre).

Statistical analysis was performed using a Student $t$ test. Differences were considered significant when $p<0.05$.

\section{Histology}

After sacrifice, the eyes of three animals were immediately immersion-fixed in Ito's solution $(2.5 \%$ glutaraldehyde, $2.5 \%$ paraformaldehyde, $10.1 \%$ picric acid in $0.1 \mathrm{M}$ cacodylate buffer, $\mathrm{pH}$ 7.2). The eyes were bisected into nasal and temporal segments, and the specimens postfixed in $1 \%$ osmium tetroxide, rinsed in cacodylate buffer, dehydrated in an ascending series of alcohols and embedded in araldite. Semithin sections were cut along a superior-inferior plane, stained with Toluidine Blue and investigated by light microscopy.

The total thickness of the neural retina and the thickness of the different retinal layers were quantitatively evaluated in the central retina. The measuring field was defined by a distance of $200 \mu \mathrm{m}$ from the optic nerve head rim, and the single-thickness measurements were obtained within the next $300 \mu \mathrm{m}$ peripherally. Since there was no statistic difference between the superior and inferior region, the data were pooled for statistical analysis using the Wilcoxon test $(p=0.01)$.

\section{Clinical investigation in humans}

\section{Dye application and surgery}

The study was approved by the local Institutional review board (IRB) and ethics committee. In a prospective, interventional, non-comparative clinical case series, eyes from consecutive patients with a $\mathrm{MH}, 13$ idiopathic, one myopic and one traumatic $\mathrm{MH}(\mathrm{n}=15)$ or epiretinal membranes (ERM, $n=3$ ) were included between April and September 2007. Macular holes were clinically classified according to Gass. ${ }^{13}$ Concomitant retinal diseases, such as very mild non-proliferative diabetic retinopathy in one patient or mild changes attributed to agerelated macular degeneration as seen in three patients, were accepted if the visual acuity (VA) of the non-study eye was not affected. All patients underwent standard 20-gauge three-port pars plana vitrectomy as described earlier. ${ }^{6}$ BBG solution $(0.5 \mathrm{~mL}, 0.25 \%$, Brilliant Peel; Fluoron GmbH, Neu-Ulm, Germany) was injected into the fluid filled globe over the macular area and washed out after a few seconds by irrigation. In patients with ERM, the dye was injected following the removal of the visible part of the ERM. The stained ILM was then grabbed using an end-gripping forceps and peeled off. A $15 \%$ hexafluoroethane $\left(\mathrm{C}_{2} \mathrm{~F}_{6}\right)$ gas mixture was used as a tamponade in $\mathrm{MH}$ cases, and patients were encouraged to keep a prone position for 5 days. Tissue removed during surgery was prepared for ultrastructural anaylsis as described previously. ${ }^{14}$ All surgeries were performed by a single surgeon $(\mathrm{CH})$, who also graded the staining of the ILM during surgery as good, weak, variable or absent.

\section{Clinical examination}

Before and after surgery, all patients underwent a complete clinical examination including measurement of best corrected VA and intraocular pressure (IOP), colour and red-free fundus photography and optical coherence tomography (OCT) (Stratus OCT, Carl Zeiss Meditec, Jena, Germany). With respect to our previous observations ${ }^{6}$ of peripheral visual field defects, we preferred manual Goldmann perimetry for the assessment of the visual field before and after surgery. However, if during followup a complete Goldmann perimetry could not be obtained, we performed an automated perimetry for the $30^{\circ}$ visual field, completed by an evaluation of the periphery by manual Goldmann perimetry.

Multifocal electroretinography (MERG) was performed in randomly selected cases $(n=14)$. Patients were seen 1 day before surgery and then in approximately 4-week intervals by one of the authors (MR). While the clinical examination, fundus photography and OCT were performed at each follow-up visit, perimetry and MERG were performed at the first postoperative examination.

Exclusion criteria were ocular diseases such as glaucoma, ischaemic optic neuropathy, any intraocular surgery within the last 60 days of the study eye, age less than 18 years and participation in other clinical investigations.

The treated eye, adverse events as well as the patient's lens status (phakic or pseudophakic), gender, age and treated eye were recorded. OCT was used to support the clinical diagnosis of a $\mathrm{MH}$ and evaluate the anatomic configuration of the macular area postoperatively and to measure retinal thickness $(\mu \mathrm{m})$ in patients with ERMs.

Statistical analysis of pre- and postoperative VA was performed using a two-tailed Wilcoxon signed ranks test for two related samples. Differences were considered significant when $p<0.05$. Patients' characteristics are summarised in table 1.

\section{RESULTS}

\section{Animal study}

\section{RGC numbers}

Whole mounts with Fluorogold-labelled retinal ganglion cells were photographed 7 days after injections of BBG and injections of balanced salt solution as controls (fig 1). Retinal ganglion cells numbers were then counted (fig 2); numbers are expressed as cell densities. In BBG injected eyes (2129 (61) cells $/ \mathrm{mm}^{2}$, mean (SEM), $\mathrm{n}=11$ ) no statistically significant differences in RGC densities were observed compared with BSS injected eyes (2202 (85), $\mathrm{n}=5, \mathrm{p}=0.43$ ).

\section{Histological evaluation}

BBG- and BSS-injected eyes showed normal retinal morphology with no signs of inflammation or degeneration throughout the central and peripheral retina. Quantification of the central retinal layers revealed normal values in the BBG-treated eyes and no statistical differences (table 2).

\section{Clinical investigation in humans}

We included 13 women and five men with a mean age of 67 years (SD 10) years in this investigation. We treated 10 right and eight left eyes. The mean follow-up up was 8 (3) weeks (range 4-14 weeks).

Over all patients, the mean VA before surgery was 20/80 (20/ 200) SD (range from 20/400 to 20/40) and increased not significantly to $20 / 60$ (20/100) SD (range from 20/250 to 20/25) 
Table 1 Clinical data of all patients

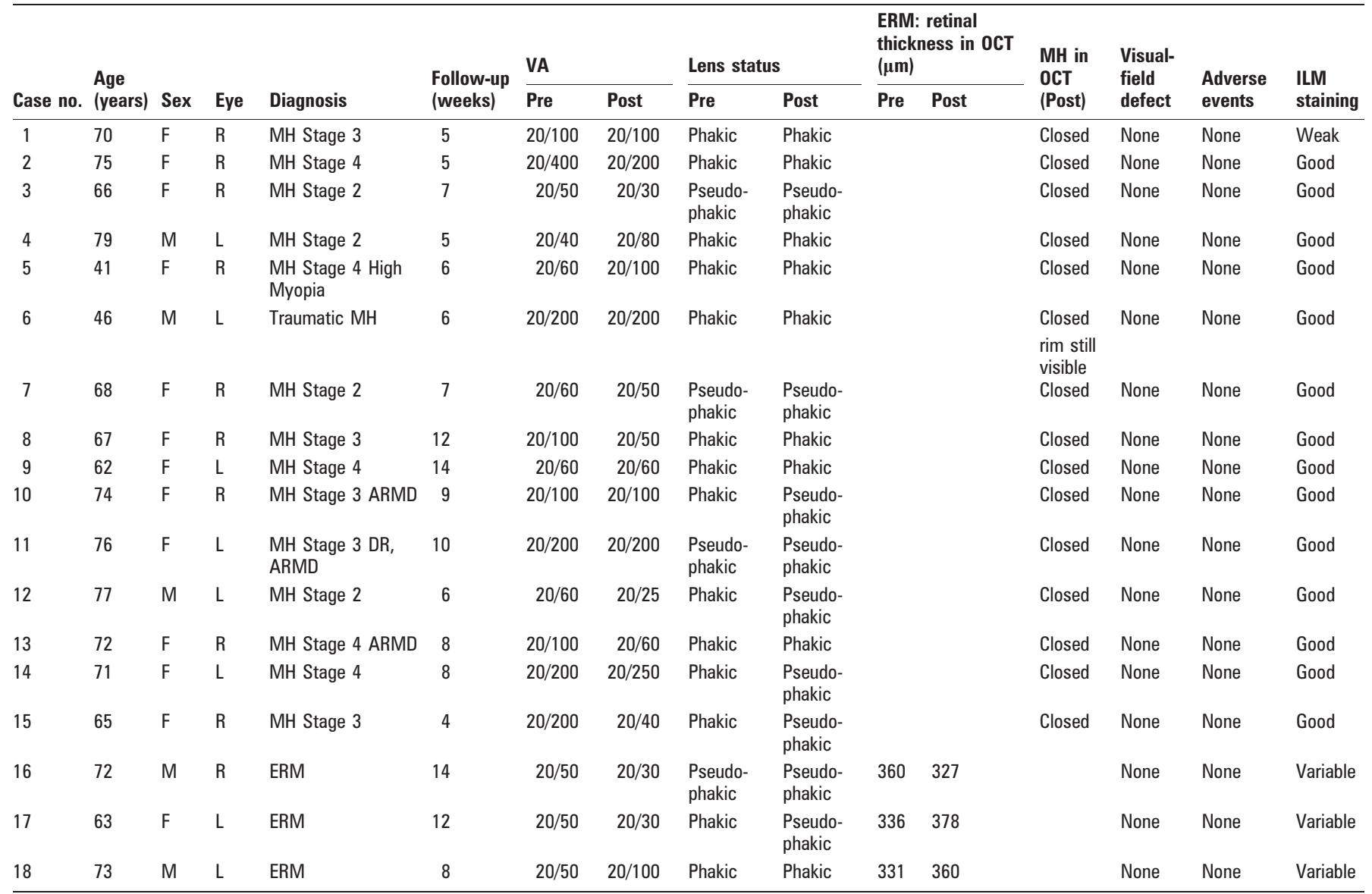

ARMD, age-related macular degeneration; DR diabetic retinopathy; ERM, epiretinal membrane; $F$, female; L, left; $M$, male; ME, macular oedema; $M H$, macular hole; Post, postoperative; Pre, preoperative; $R$, right; VA, visual acuity.

after surgery $(p=0,094)$. Looking at the group of $\mathrm{MH}$ patients, the mean VA improved not significantly $(p=0,090)$ from $20 /$ 100 (20/200) SD (range from 20/400 to 20/40) to 20/60 (20/ $100)$ SD (range from $20 / 250$ to $20 / 25$ ). VA improved in 10 eyes (56\%, 8/15 MH patients, 2/3 ERM patients), was unchanged in four eyes (22\%, all $\mathrm{MH}$ patients) and decreased in four eyes (28\%, 3/15 MH, 1/3 ERM). In MH patients, the lack of visual improvement was partially related to the pathogenesis of the $\mathrm{MH}$ such as myopic and traumatic $\mathrm{MH}$ (patient 5 and 6) or may be attributed to concomitant diseases such as diabetic retinopathy and age-related macular degeneration (ARMD) (patients 10 and 11) that might have had an influence on the development of visual acuity. Two of the ERM patients (patients 16 and 17) developed a macular oedema seen shortly after surgery with an increase in retinal thickness to $412 \mu \mathrm{m}$ and $630 \mu \mathrm{m}$ respectively and a visual acuity of $20 / 40$ and $20 /$ 500. They were therefore treated by retrobulbar steroid injection, which led to a significant improvement of both retinal thickness $(327 \mu \mathrm{m}$ and $378 \mu \mathrm{m})$ and visual acuity (20/30 each) (table 1$)$. The IOP was in normal range at any time.

In five eyes, surgery had been performed as a combined procedure of vitrectomy and cataract extraction with posterior chamber lens implantation. Nine eyes were pseudophakic at the last follow-up visit.

All MHs were completely closed as shown by optic coherence tomography or clinical investigation after the first surgical intervention. In one patient (patient 6) with a large traumatic $\mathrm{MH}$, the rim of the was postoperatively attached but still visible.
Figure 1 Fluorogold-labelled retinal ganglion cells photographed 7 days after injections of Brilliant Blue $G(A)$ and injections of balanced salt solution $(B)$ as controls.
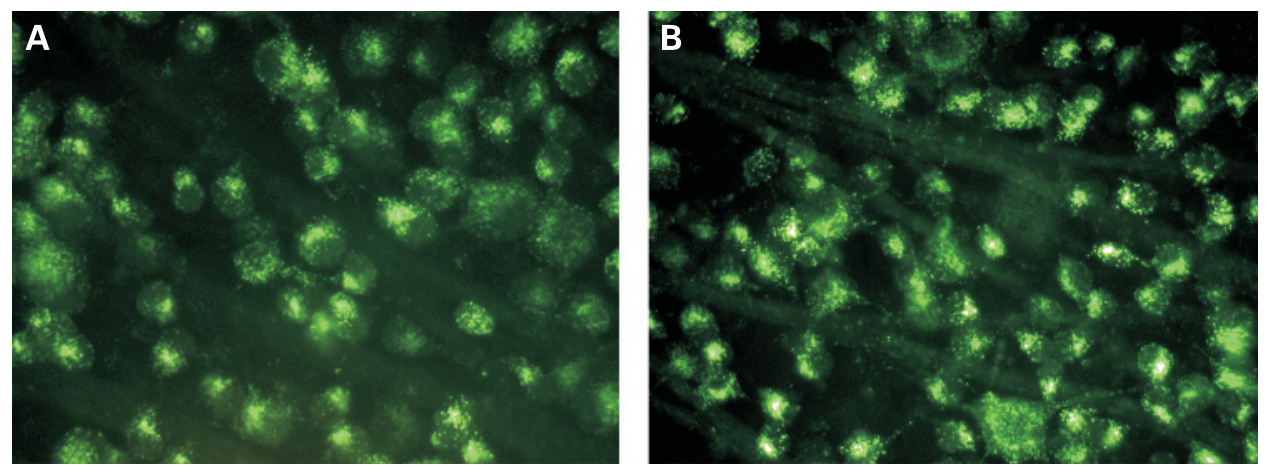


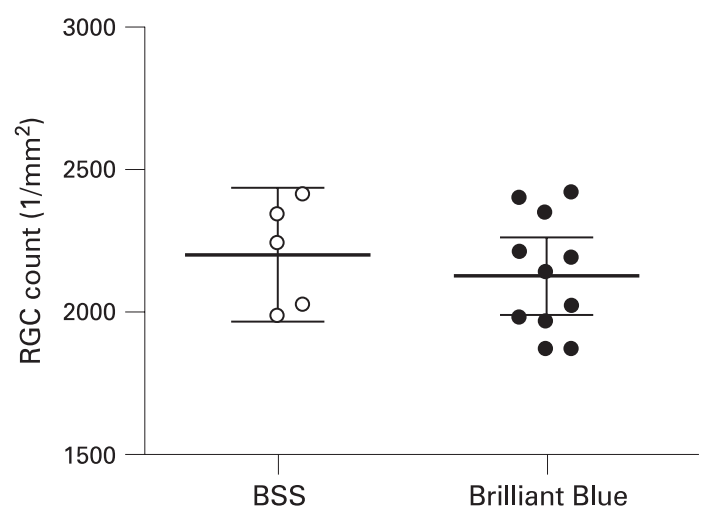

Figure 2 Retinal ganglion cell (RGC) numbers counted and numbers expressed as cell densities. In Brilliant Blue G-injected eyes (2129 (61) cells $/ \mathrm{mm}^{2}$, mean (SEM), $\mathrm{n}=11$ ) no statistically significant differences in retinal ganglion cell densities were observed compared with balanced salt solution (BSS)-injected eyes (2202 (85), $n=5, p=0.43$ ).

We did not observe any visual field defects or any abnormalities in the other functional tests such as MERG that might indicate toxic effects of the dye. Fundus photographs did not show any abnormal alterations of the retinal pigment epithelium or defects of the nerve fibre layer seen in red-free images. There were no systemic side effects.

Staining of the ILM during $\mathrm{MH}$ surgery was successful in 15 cases and allowed for an easy and controlled removal of the ILM with no haemorrhages of the retinal surface indicating surgical trauma (fig 3). In patients with ERMs, the staining of the ILM was variable. This was most likely attributed to the fact that the dyes had been injected after removal of the ERM, indicating that the ILM is peeled off along with the ERM in a variable extent. Transmission electron microscopy confirmed the presence of the ILM in all processed specimens $(n=18)$. The complete data on our patients are presented in table 1.

\section{Ultrastructure of removed membranes}

The ultrastructural analysis revealed fragments of the ILM in all specimens. There was a variable amount of epiretinal cellular proliferation seen at the vitreal side of the ILM without any cellular damage. The nuclei and cytoplasmatic elements such as mitochondriae or golgi complexes were well preserved. At the retinal side of the ILM, no relevant amount of retinal debris was seen (fig 4)

\section{DISCUSSION}

There is common agreement that toxic effects of any new dye suggested to assist intraocular surgery need to be carefully ruled out prior to its routine use in humans. Shortly after ICG had been recommended for ILM peeling, ${ }^{3}$ a less favourable functional outcome and a high incidence of peripheral visual field defects $^{6}{ }^{15} 16$ led to a still ongoing discussion on potential toxic effects of ICG which may be related to its photosensitising properties. ${ }^{17} 18$ The safety margin of ICG is unclear, and some authors did not observe any dye-related complications. ${ }^{19} 20 \mathrm{~A}$ new and promising dye to visualise the ILM may be BBG. ${ }^{10}{ }^{11}$ First, experimental and clinical data, obtained in animals and humans, addressed the toxicity of BBG following subretinal injection $^{9}$ and revealed a good biocompatibility of BBG in contrast to control dyes including ICG and TB. An experimental study exposing glial cells to ICG and BBG disclosed ICG toxicity as seen by the induction of apoptosis, involving induction of the caspase cascade through p38 mitogen-activated protein kinase phosphorylation while BBG did not cause apoptosis and therefore could be considered a safer adjuvant. ${ }^{21}$ In our own study, the dye was injected intravitreally and therefore had contact to the retinal surface but not the subretinal space as in previous investigations. ${ }^{9}$ The analysis of retinal ganglion cell numbers showed that intravitreal BBG did not lead to a significant change in RGC numbers in rat retina in vivo. It is important to note that our experimental setting to assess retinal toxicity does not resemble the situation in vitreoretina surgery exactly. First, the dye is injected into the vitreous gel, which is usually removed in human eyes before applying the dye during vitreoretinal surgery, which could lead to a higher local dose of the dye during vitreoretinal surgery. Second, the exposure time of 7 days in the rat eye is much longer than the intended application in humans, where the dye is washed out immediately by irrigation. However, we chose this setting, as it seems reasonable to assume that a dye leading to no visible toxic effect after 1 week will not show such an effect after 1 minute as well. Although the extended exposure time as chosen here offers additional information on the safety margin of BBG, one has to keep in mind that toxicity studies in animals may not resemble all possible toxic effects in a clinical situation in humans. Therefore, long-term side effects seen in humans during prospective clinical trials cannot be ruled out.

Following the positive results of our investigation in the animal, we decided to use the dye in humans as well. BBG was applied to assist $\mathrm{MH}$ surgery and surgery for epiretinal membranes. In $\mathrm{MH}$ surgery, the dye provided a excellent contrast of the ILM and allowed for a complete removal of the ILM. We did not observe any functional deficits in terms of visual field defects in our patient as seen following the use of ICG. ${ }^{6}$ No abnormalities of the RPE were seen clinically and in fundus photographs, although the dye may very well have come into contact with the RPE through the $\mathrm{MH}$. The closure rate was comparable with our results obtained without dye assistance. $^{22}$ VA improvement in $\mathrm{MH}$ patients was less pronounced compared with our previous studies where we observed a mean VA of 20/40 postoperatively. ${ }^{22}$ From our point of view, this is related to the relatively short follow-up in some patients, patient selection including concomitant diseases such as high myopia, trauma, ARMD or DR and the lens status. Despite the relatively short follow-up in some cases, conclusions on the safety of BBG can be drawn as in previous investigations on ICG adverse events such as visual field defects becoming apparent already at the first follow-up visit. ${ }^{6}$ Of note, we did not observe any defects of the nerve fibre layer seen in red-free

Table 2 Quantification of the central retinal layers

\begin{tabular}{|c|c|c|c|c|c|c|c|c|c|}
\hline & Total $(\mu \mathrm{m})$ & NFL $(\mu \mathrm{m})$ & GCL $(\mu \mathrm{m})$ & IPL $(\mu \mathrm{m})$ & INL (rows) & $\mathrm{OPL}(\mu \mathrm{m})$ & ONL (rows) & PR-IS $(\mu \mathrm{m})$ & PR-0S $(\mu \mathrm{m})$ \\
\hline OD, mean (SD) & $223(7)$ & $8.5(2.9)$ & $12.6(2.4)$ & $67(7)$ & $5.2(0.4)$ & $6.4(0.9)$ & $9.8(0.4)$ & $17(3)$ & $26(3)$ \\
\hline OS, mean $(S D)$ & $216(8)$ & $8.9(1.4)$ & $13.2(2.5)$ & $64(4)$ & $5.2(0.4)$ & $7.5(0.9)$ & $9.8(0.4)$ & $15(3)$ & $24(2)$ \\
\hline
\end{tabular}

Quantification of the central retina in Brilliant Blue G (OS) and BSS (OD, control) injected eyes

$\mathrm{GCL}$, ganglion-cell layer; INL, inner nuclear layer; IPL, inner plexiform layer; NFL, nerve-fibre layer; ONL, outer nuclear layer; OPL, outer plexiform layer; PR-IS, photo receptor inner segment; PR-OS, photoreceptor outer segment; Total, whole thickness. 
Figure 3 Peeling of the Brilliant Blue Gstained internal limiting membrane in a patient with a traumatic macular hole (patient 6).
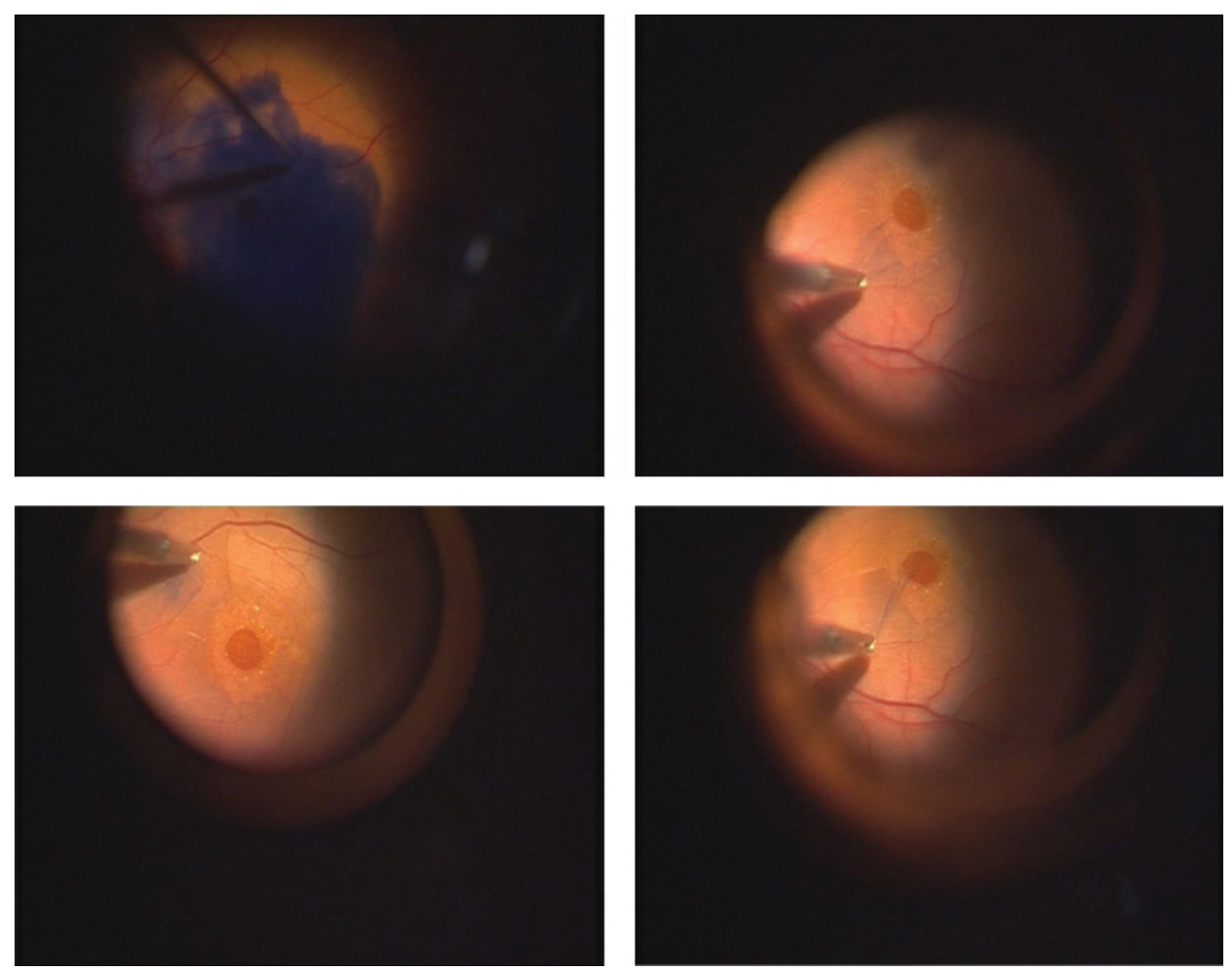

fundus photographs as described in previous publications on ILM peeling without any dye. ${ }^{23}$ These asymptomatic defects had been additionally confirmed by microperimetry as central visual field defects and were very likely correlated to a mechanical, focal trauma of the innermost retinal layers by ILM peeling. One may conclude therefore that BBG-assisted ILM peeling is less traumatic to the nerve fibre layer.

During surgery for epiretinal membranes BBG was injected following the removal of the visible epiretinal membrane using an end-gripping forceps in order to visualise fragments of the ILM

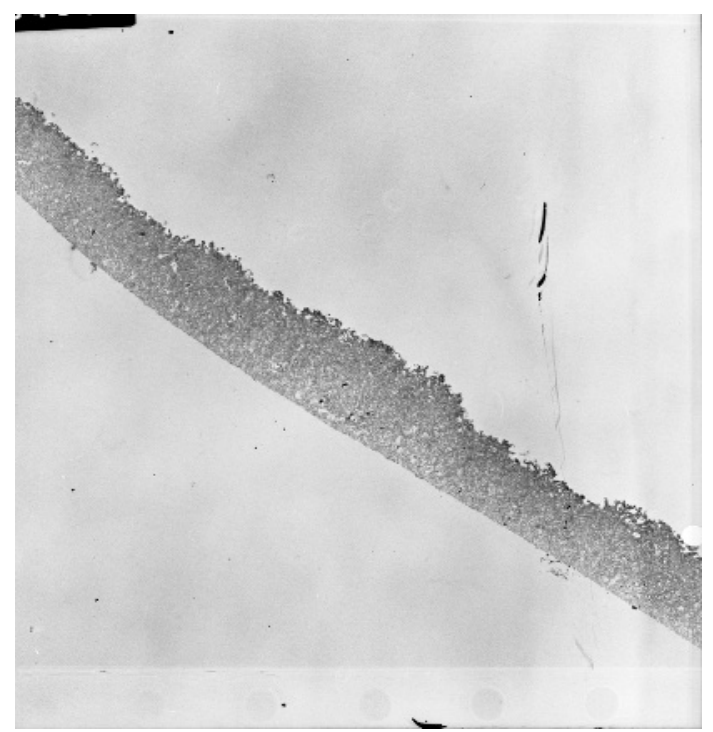

Figure 4 Ultrastructure of the internal limiting membrane removed during Brilliant Blue G-assisted macular hole surgery. Note the absence of retinal debris at the retinal (rough) surface of the internal limiting membrane. Magnification $1800 \times$. which were not removed along with the epiretinal membrane. As a consequence, the staining pattern was not as homogenous as seen during $\mathrm{MH}$ surgery, but provided a more patchy aspect. As during $\mathrm{MH}$ surgery, the ILM could be easily removed without damage to the underlying retina and with no toxic effects of the dye seen postoperatively. However, during all surgical procedures, the surgeon noted an increased rigidity of the stained ILM compared with previous surgeries without BBG application.

Our data underline the good biocompatibility of BBG and its applicability and safety for the use in humans. BBG provides a sufficient and selective staining of the ILM. No retinal toxicity related to BBG was observed in our animal study and our shortterm clinical investigation in humans. As mentioned earlier, we feel that it is of great importance to collect as much safety data as possible on a new dye suggested for intraocular surgery in humans. The long-term safety of this novel dye will have to be evaluated in larger patient series and a longer follow-up.

Acknowledgements: The authors do not have any commercial or proprietary interest in the drugs tested in this study. We thank R Scheler, Technical Assistant, for excellent technical support.

\section{Competing interests: None.}

Ethics approval: The study was approved by the local Institutional review board (IRB) and ethics committee.

\section{REFERENCES}

1. Kadonosono K, Itoh N, Uchio E, et al. Staining of the internal limiting membrane in macular hole surgery. Arch Ophthalmol 2000;118:1116-18.

2. Feron EJ, Veckeneer M, Parys-Van Ginderdeuren R, et al. Trypan blue staining of epiretinal membranes in proliferative vitreoretinopathy. Arch Ophthalmol 2002;120:141-4.

3. Haritoglou C, Strauss R, Priglinger SG, et al. Delineation of the vitreous and posterior hyaloid using bromophenol blue. Retina 2008;28:333-9.

4. Gass CA, Haritoglou C, Schaumberger M, et al. Functional outcome of macular hole surgery with and without indocyanine green-assisted peeling of the internal limiting membrane. Graefe's Arch Clin Exp Ophthalmol 2003;241:716-20.

5. Uemura A, Kanda S, Sakamoto Y, et al. Visual field defects after uneventful vitrectomy for epiretinal membrane with indocyanine green-assisted internal limiting membrane peeling. Am J Ophthalmol 2003;136:252-7. 
6. Haritoglou C, Gandorfer A, Gass CA, et al. Indocyanine green-assisted peeling of the internal limiting membrane in macular hole surgery affects visual outcome: A clinicopathologic correlation. Am J Ophthalmol 2002;134:836-41.

7. Ferencz M, Somfai GM, Farkas A, et al. Functional assessment of the possible toxicity of indocyanine green dye in macular hole surgery. Am J Ophthalmol 2006;142:756-70.

8. Lesnik Oberstein SY, Mura M, Tan SH, et al. Heavy trypan blue staining of epiretinal membranes: an alternative to infracyanine green. Br J Ophthalmol 2007;91:955-7.

9. Ueno $\mathbf{A}$, Hisatomi $\mathrm{T}$, Enaida $\mathrm{H}$, et al. Biocompatibility of brilliant blue $\mathrm{G}$ in a rat model of subretinal injection. Retina 2007;27:499-504.

10. Enaida $\mathbf{H}$, Hisatomi $\mathrm{T}$, Hata $\mathrm{Y}$, et al. Brilliant blue $\mathrm{G}$ selectively stains the internal limiting membrane/brilliant blue G-assisted membrane peeling. Retina 2006;26: 631-6.

11. Enaida $\mathbf{H}$, Hisatomi $\mathrm{T}$, Goto $\mathrm{Y}$, et al. Preclinical investigation of internal limiting membrane staining and peeling using intravitreal brilliant blue G. Retina 2006;26 623-30.

12. Schuettauf $\mathbf{F}$, Haritoglou $C$, May CA, et al. Administration of novel dyes for intraocular surgery: an in vivo toxicity animal study. Invest Ophthalmol Vis Sci 2006;47:3573-8.

13. Gass JD. Idiopathic senile macular hole. Its early stages and pathogenesis. Arch Ophthalmol 1988:106:629-39.

14. Schumann RG, Schaumberger MM, Rohleder M, et al. Ultrastructure of the vitreomacular interface in full-thickness idiopathic macular holes: a consecutive analysis of 100 cases. Am J Ophthalmol 2006;141:1112-19.
15. Ando F, Yasui O, Hirose $\mathrm{H}$, et al. Optic nerve atrophy after vitrectomy with indocyanine green-assisted internal limiting membrane peeling in diffuse diabetic macular edema. Adverse effect of ICG-assisted ILM peeling. Graefes Arch Clin Exp Ophthalmol 2004:242:995-9.

16. Tognetto D, Grandin R, Sanguinetti G, et al. Internal limiting membrane removal during macular hole surgery: results of a multicenter retrospective study. Ophthalmology 2006;113:1401-10.

17. Haritoglou C, Priglinger SG, Gandorfer A, et al. Histology of the vitreoretinal interface after indocyanine green staining of the ILM with illumination using a halogen and xenon light source. Invest Ophthalmol Vis Sci 2005;46:1468-72.

18. Peters S, Altvater A, Bopp S, et al. Systematic evaluation of ICG and trypan blue related effects on ARPE-19 cells in vitro. Exp Eye Res 2007;85:880-9.

19. Lai MM, Williams GA. Anatomical and visual outcomes of idiopathic macular hole surgery with internal limiting membrane removal using low-concentration indocyanine green. Retina 2007:27:477-82.

20. Ullern M, Dubreuil F, Nourry H, et al. Macular hole surgery with and without infracyanine-green-guided removal of the internal limiting membrane. J Fr Ophtalmol 2007; $30: 53-7$.

21. Kawahara S, Hata Y, Miura M, et al. Intracellular events in retinal glial cells exposed to ICG and BBG. Invest Ophthalmol Vis Sci 2007;48:4426-32.

22. Reiniger IW, Gass CA, Schaumberger M, et al. Long-term functional results after macular hole surgery. Results of a prospective study. Ophthalmologe 2006; 103:501-5.

23. Haritoglou C, Ehrt 0, Gass CA, et al. Paracentral scotomata: a new finding after vitrectomy for idiopathic macular hole. Br J Ophthalmol 2001;85:231-3. 


\section{An in vivo evaluation of Brilliant Blue $G$ in animals and humans}

M Remy, S Thaler, R G Schumann, et al.

Br J Ophthalmol 2008 92: 1142-1147

doi: 10.1136/bjo.2008.138164

Updated information and services can be found at:

http://bjo.bmj.com/content/92/8/1142.full.html

\begin{tabular}{|c|c|}
\hline \multirow[b]{2}{*}{ References } & These include: \\
\hline & $\begin{array}{l}\text { This article cites } 23 \text { articles, } 5 \text { of which can be accessed free at: } \\
\text { http://bjo.bmj..com/content/92/8/1142.full.html\#ref-list-1 }\end{array}$ \\
\hline \multirow[b]{2}{*}{$\begin{array}{l}\text { Email alerting } \\
\text { service }\end{array}$} & $\begin{array}{l}\text { Article cited in: } \\
\text { http://bjo.bmj.com/content/92/8/1142.full.html\#related-urls }\end{array}$ \\
\hline & $\begin{array}{l}\text { Receive free email alerts when new articles cite this article. Sign up in } \\
\text { the box at the top right corner of the online article. }\end{array}$ \\
\hline \multirow{2}{*}{$\begin{array}{l}\text { Topic } \\
\text { Collections }\end{array}$} & Articles on similar topics can be found in the following collections \\
\hline & $\begin{array}{l}\text { Retina (1293 articles) } \\
\text { Ophthalmologic surgical procedures (1024 articles) }\end{array}$ \\
\hline
\end{tabular}

Notes

To request permissions go to:

http://group.bmj.com/group/rights-licensing/permissions

To order reprints go to:

http://journals.bmj.com/cgi/reprintform

To subscribe to BMJ go to:

http://group.bmj.com/subscribe/ 